\title{
Investigation of E2, E5, and E6 gene expression and DNA in situ fragmentation findings associated with progressive and regressive changes in benign neoplastic cutaneous lesions arising naturally from bovine papillomavirus- 1 infection
}

\author{
MEHMET ERAY ALCIGIR, SEVIL ATALAY VURAL, MEHMET OZKAN TIMURKAN* \\ Department of Pathology, Faculty of Veterinary Medicine, Ankara University, 06110, Diskapi-Ankara, Turkey \\ *Department of Virology, Faculty of Veterinary Medicine, Ataturk University, 25240, Yakutiye-Erzurum, Turkey
}

Alcıgır M. E., Atalay Vural S., Tımurkan M. O.

Investigation of E2, E5, and E6 gene expression and DNA in situ fragmentation findings associated with progressive and regressive changes in benign neoplastic cutaneous lesions arising naturally from bovine papillomavirus-1 infection

Summary

This study investigated the progressive and regressive phases of bovine papillomavirus (BPV)-1-induced papilloma and fibropapilloma in terms of the distribution of viral antigenicity; E2, E5, and E6 gene activity and expression; and apoptosis (evaluated using DNA in situ fragmentation). All samples from 74 bovine cases of cutaneous lesions with suspected neoplastic changes collected from different skin areas were evaluated. The lesions were diagnosed as papilloma or fibropapilloma using routine hematoxylin-eosin and Mallory's trichrome staining protocols. Feulgen reactions suggested a possible viral entity, and BPV-1 antigenicity was immunohistochemically observed in 22 cases. $E$ gene expression in neoplastic tissues was evaluated using chromogenic in situ hybridization and reverse-transcription polymerase chain reaction, and the TUNEL method was used to evaluate DNA in situ fragmentation. Differences between progressive and regressive phases in these lesions were analyzed. Ultimately, the number of cases in the progressive phase (18) and regressive phase (4) were determined. Although E2 and E5 gene expression was observed in both phases, E6 gene distribution differed from the other $\mathbf{E}$ genes distribution in the progressive phase. $\mathbf{E} 5$ gene expression appeared stronger in oncogenic fibrocytes and fibroblasts, whereas E2 and E6 gene expression was weaker in epidermal cells and cells in the regressive phase. Apoptosis, considered the best indicator of neoplastic regression, was found to be associated with the regressive phase but was observed insufficiently in epidermal and dermal samples of the progressive-phase cases. In conclusion, this work provides significant insights on the molecular and immunopathological features of BPV-1.

Keywords: apoptosis, BPV-1, E genes, progressive and regressive phases

Bovine papilloma and fibropapilloma are among the benign neoplastic changes observed in cattle (1). Notably, several types of papillomavirus have been implicated in the etiology of viral-induced papillomas (2), including bovine papillomaviruses (BPVs) -1 to -6 (5). More recently, the family Papillomaviridae has been expanded to include 13 members (BPV-1 to -13), and BPV infection has been found to not only affect cattle but also humans and several other domestic and wild animal species $(24,25)$.
Papilloma and/or fibropapilloma are characterized by progressive and regressive phases. The progressive phase almost always follows the regressive phase in immunocompetent animals, mounting an immune response to BPV-1 infection, during which mononuclear cells, lymphocytes, and macrophages are activated to protect host cells from the virus (6). The progressive phase is characterized by hyperplastic stratum spinosum and acanthosis, hyperkeratosis, apoptosis, and occasional inflammatory reactions; 
keratohyaline granules and prominent stromal proliferation may also be observed. Stromal proliferation is particularly predominant in fibropapillomas. The regressive phase is characterized by thinning of the stratum spinosum along with concomitant thickening of the stratum corneum, necrosis of compact collagenous tissues, and vascular degeneration in the stratum spinosum (12).

Currently, the molecular aspects of BPV-1 have not been fully elucidated. However, previous reports have yielded the following information. The E2 gene product detects and attaches to replication sites. The E5 gene product exerts its oncogenic effects during the progressive phase by destroying the cellular mechanisms that control growth and development. The E5 and E6 gene products act synergistically during the progressive phase to promote cellular transformation. In addition, the E6 gene product was found to induce apoptotic sensitivity in neoplastic cells, particularly during a prolonged regression phase $(4,17,19)$.

This study aimed to detect and compare the distribution of BPV-1 antigenicity, determine the E genes with the most important roles in etiopathogenesis, and evaluate DNA in situ fragmentation results associated with progressive and regressive changes in cases of papilloma and fibropapilloma. In the epidermis, viral E2 and E5 gene activities were predominantly found to correlate with the BPV-1 entity and distribution; however, these genes had less of an effect on stromal compartments in the neoplastic epidermis. In addition, the decreases in E2 gene expression and apoptosis were less marked in the regressive phase compared with those in the progressive phase. Finally, the E5 gene had a stronger effect than that expected in the connective tissue compartment during the regressive phase, whereas the E6 gene had no such effect.

\section{Material and methods}

Macroscopic examination. The study was approved and confirmed by the Local Ethical Committee at Ankara University, Ankara, Turkey (number: 53184147/56050, date: 10.21.2013). Neoplastic masses were collected from 74 cattle aged 2-3 years in herds in different provinces (Ankara, Bursa, Samsun) of Turkey. Twenty-two BPV-1-positive cattle were identified (six females, 16 males). The masses were evaluated according to size, weight, elasticity, color, and appearance of the outer and cut sections. Twelve positive cases included solid masses over several regions of the body, including the ear $(\mathrm{n}=2)$, eyelid $(\mathrm{n}=1)$, neck $(n=1)$, hind $\operatorname{limb}(n=3)$, caudal abdominal region $(n=1)$, and mammary region $(n=4)$. Ten cases featured multiple lesions in those regions.

Histopathological examination. All collected samples were processed routinely after fixation in $10 \%$ buffered formalin. The samples were embedded in paraffin and cut into $4-\mu \mathrm{m}$-thick sections. Sections from samples diagnosed as papilloma and fibropapilloma using hematoxylin-eosin and Mallory's trichrome staining were further subjected to Feulgen staining to determine the presence of viral DNA.
Immunoperoxidase method. A streptavidin-biotin complex peroxidase/Strept ABC-P method was used for the immunohistochemical analysis. Sections were deparaffinized and hydrated in a graded alcohol series. After treatment with $0.1 \%$ trypsin for $15 \mathrm{~min}$ at $37^{\circ} \mathrm{C}$, endogenous peroxidase activity in the tissues was blocked with a 3\% (v/v)-hydrogen peroxide $\left(\mathrm{H}_{2} \mathrm{O}_{2}\right)$-methanol solution for $15 \mathrm{~min}$. After a 30 min nonspecific protein blocking step, sections were incubated with primary serum (monoclonal mouse anti-BPV-1 serum; AA182-190; Antibodies Online, Aachen, Germany) for $1 \mathrm{~h}$ at the same temperature. Thereafter, the sections were incubated sequentially with linker and secondary antibodies (a biotinylated antibody and streptavidin-horseradish peroxidase-labeled antibody, respectively; RE7110-K; Novocastra/Leica Biosystems, Nussbach, Germany) for $30 \mathrm{~min}$ each. Prior to that step, the sections were subjected to two to five min washes in phosphate-buffered saline (PBS) after all steps except protein blocking. Finally, reactions were visualized using the chromogen diaminobenzidine (DAB), with Gill's hematoxylin as a counterstain.

Stained sections were passed through an alcohol-xylol series and mounted with nonaqueous mounting medium. The negative controls included sections from nonlesionedbovine skin samples and those stained with PBS instead of primary serum. The positive controls included sections of BPV-1 (viral capside protein, L1 and L2) positive tissues from urinary bladder tumor tissue samples archived at the Department of Pathology; these sections were stained as described above for experimental samples.

TUNEL in situ detection of apoptosis. To detect DNA fragmentation, the terminal deoxynucleotidyl transferase (TdT)-mediated nick end-labeling (TUNEL) method was used according to the instructions provided with the biotin-labeled In Situ Cell Death Detection Kit (number 11684817910; Roche, Basel, Switzerland). The staining procedure was identical to that for immunohistochemistry up to the protein blocking step. Subsequently, a TUNEL mixture comprising the enzyme and labeling solution was placed on the sections, which were incubated for $1 \mathrm{~h}$ at the same temperature. The sections were then incubated with the POD converter for $30 \mathrm{~min}$. All subsequent steps were identical to those described above for the immunoperoxidase method. For negative control sections, the labeling solution alone was used instead of the TUNEL mixture.

Chromogenic in situ hybridization (CISH). To detect viral E gene activity, tissue sections were deparaffinized, hydrated, and subjected to staining with a CISH kit (ZytoFast PLUS CISH Implementation Kit HRP-DAB, T-1063-40; Zyto Vision GmbH, Bremerhaven, Germany). Sections were first incubated in a $3 \% \mathrm{H}_{2} \mathrm{O}_{2}$ solution for $10 \mathrm{~min}$, followed by pepsin at $37^{\circ} \mathrm{C}$ for $5 \mathrm{~min}$. The sections were then pretreated with ethylenediaminetetraacetic acid (EDTA) at $95^{\circ} \mathrm{C}$ for $15 \mathrm{~min}$. After washing with Trisbuffered saline (TBS), the sections were passed through a graded series of alcohols and air-dried. Oligoprobes* specific to the E2, E5, and E6 genes of BPV-1 (designed and synthesized by Metabion International AG, Germany; Tab. 1) were diluted to the appropriate concentration and applied to the samples. The stained sections were mounted with a frame and coverslip, (volume: $125 \mu$, dimensions: $1.7 \times 2.8 \mathrm{~cm}$; AB-0578; Thermo Scientific, Waltham, MA, 
Tab. 1. *Base sequences of DNA oligoprobes of digoxigenin labelled E2, E5 and E6 of BPV-1

\begin{tabular}{|c|c|c|}
\hline Gene & Base Sequence of the oligoprobes & Base size \\
\hline E2 & 5'-Digoxigenin-CGT-TGC-CGC-TCG-GGG-TCA-AAC-CGT-CGG-TGC-TCG-AAA-CGC-CCT-3' & 46 base \\
E5 & 5'-Digoxigenin-CTG-TTC-TTA-CTC-TTG-TTT-TTT-CTT-GTA-TAC-TGG-GAT-CAT-TTT-GAG-3' & 45 base \\
E6 & 5'-Digoxigenin-GTT-GTA-ATT-CGG-GAA-GGC-TGT-AGA-TAT-GGT-GCA-TGT-ACC-ATT-TGT-3' & 45 base \\
\hline
\end{tabular}

Tab. 2. **Forward and Reverse Primers, the annealing degrees and PCR product sizes

\begin{tabular}{|c|c|c|c|c|}
\hline \multirow{2}{*}{ Gene } & Forward & Reverse & $\begin{array}{c}\text { Annealing } \\
\left({ }^{\circ} \mathrm{C}\right)\end{array}$ & $\begin{array}{c}\text { Product } \\
\text { size }\end{array}$ \\
\hline E2 & CCATGGTCTTTGCTTGACAC & CTGCCAGCCGTCCTCTGT & 55 & $170 \mathrm{bp}$ \\
E5 & TGCCAAATCTATGGTTTCTATTG & AAGAGTAAGAACAGTAACAGCAGCA & $\mathbf{5 8}$ & $\mathbf{7 5} \mathrm{bp}$ \\
\hline E6 & ATTGCATGGCAAAACACTTG & GCTCTGGTTTTGCAGAAAGG & $\mathbf{5 2}$ & $\mathbf{1 1 9} \mathrm{bp}$ \\
\hline
\end{tabular}

USA), followed by denaturation at $75^{\circ} \mathrm{C}$ for $5 \mathrm{~min}$ and hybridization at $55^{\circ} \mathrm{C}$ for $1 \mathrm{~h}$ in a thermal cycler (Omnislide; Thermo Scientific).

After removing the coverslip, the sections were washed twice with $1 \times \mathrm{TBS}$ at $55^{\circ} \mathrm{C}$ for $5 \mathrm{~min}$, followed by incubation with mouse anti-digoxigenin anti-serum at $37^{\circ} \mathrm{C}$ for $30 \mathrm{~min}$. After three $1 \mathrm{~min}$ washes with TBS, an anti-mouse HRP polymer solution was placed on the sections, followed by incubation at the same temperature and for the same duration. The sections were then subjected to three 2 min washes in PBS and incubated with DAB for 10 min. Following washing in distilled water, the sections were counterstained with a nuclear blue solution for $10 \mathrm{~min}$, washed in tap water for $5 \mathrm{~min}$, dehydrated in a graded alcohol-xylol series, and mounted with entellan. For the negative and positive DNA/RNA control samples, the probes provided with the kit (ZytoVision, ZytoFast PLUS CISH Implementation Kit HRP-DAB, T-1063-40) were applied to the tissue samples; all other steps were as described above.

All histopathological, immunohistochemical (for BPV-1 and DNA in situ fragmentation), and CISH results were observed with a light microscope (Leica DM-4000B) and illustrated (Leica DFC-280). The staining results in 10 highpowered fields (HPFs) were scored as follows: $(-)$, no staining; (+) $10 \%-30 \%$ positivity; $(++), 30 \%-50 \%$ positivity; and $(+++),>50 \%$ positivity. A mean value was calculated from the total number of positive samples in all fields.

Reverse Transcriptase Polymerase Chain Reaction (RT-PCR). To evaluate gene expression in the tissue, collected samples $(1 \mathrm{~g})$ stored at $-80^{\circ} \mathrm{C}$ were thawed and homogenized at 2,000 rpm (TissueLyser LT; Qiagen, Hilden, Germany) for $5 \mathrm{~min}$ in $500 \mu 1$ of PBS. Supernatants were collected by centrifugation at 3,000 rpm for $5 \mathrm{~min}$. For extraction, a viral nucleic acid extraction kit (Vivantis, Selangor Darul Ehsan, Malaysia) was used according to the manufacturer's instructions provided. For reverse transcription, an appropriate reverse transcription enzyme (Thermo Fisher Scientific, Bremen, Germany) was used kullanıldı. cDNA analysis was performed in two steps. In the first step, $6 \mu 1$ of template, $6 \mu 1$ of RNase/DNase-free water, and $1 \mu 1$ of random hexamers were cycled at $70^{\circ} \mathrm{C}$ for $5 \mathrm{~min}$. In the second step, each sample was mixed with $4 \mu 1$ of $5 \times$ RT-Buffer, $2 \mu 1$ of dNTP, and $1 \mu 1$ of reverse transcriptase; these samples were incubated in a thermal cycler at $25^{\circ} \mathrm{C}$ for $10 \mathrm{~min}, 37^{\circ} \mathrm{C}$ for $1 \mathrm{~h}$, and $70^{\circ} \mathrm{C}$ for $5 \mathrm{~min}$. For PCR, Taq
DNA polymerase (Thermo Fisher Scientific), specific primers for E2, E5, and E6* (Metabion International AG), $3 \mu \mathrm{l}$ of template, and PCR compounds were combined in thermal cycler tubes for synthesis and subjected to the following program in a thermal cycler (Veriti; Applied Biosystems, Foster City, CA, USA): a 5 min denaturation step at $94^{\circ} \mathrm{C} ; 35$ cycles of $94^{\circ} \mathrm{C}$ for $1 \mathrm{~min}$, annealing temperature** and extension at $72^{\circ} \mathrm{C}$ for $1 \mathrm{~min}$; and a final extension step at $72^{\circ} \mathrm{C}$ for $10 \mathrm{~min}$. To detect amplicons, an agarose gel was prepared, stained with ethidium bromide (Sigma, Munich, Germany), and visualized under UV transillumination (Vilber Lourmat, Collégien, France). The sample sizes are listed in Tab. 2.

\section{Results and discussion}

Macroscopic findings. The masses from the 22 positive cases were collected from different areas of the body and measured $0.5-4 \mathrm{~cm}$ in diameter. All masses had a cauliflower-like appearance. All cut sections were homogenous and grayish-white in color, particularly the cases with suspected fibropapilloma (Fig. 1). Moreover, the epidermal and stromal growth was most remarkable in cases suspected to be progressive-phase, despite the thin epidermal structure.

Microscopic findings. Mild (6/18), moderate (5/18), or strong $(7 / 18)$ hyperkeratosis, parakeratosis $(1 / 18)$, or both $(8 / 18)$ were observed in the 18 progressive papilloma and fibropapilloma cases. Similarly, mild $(5 / 18)$, moderate $(9 / 18)$, or strong $(8 / 18)$ hyperplasia and acanthosis were observed. Acute cell swelling $(13 / 18)$ and vacuolar degeneration $(7 / 18)$ were present, particularly above the stratum spinosum. Although two cases did not exhibit degeneration, mild (13/18),

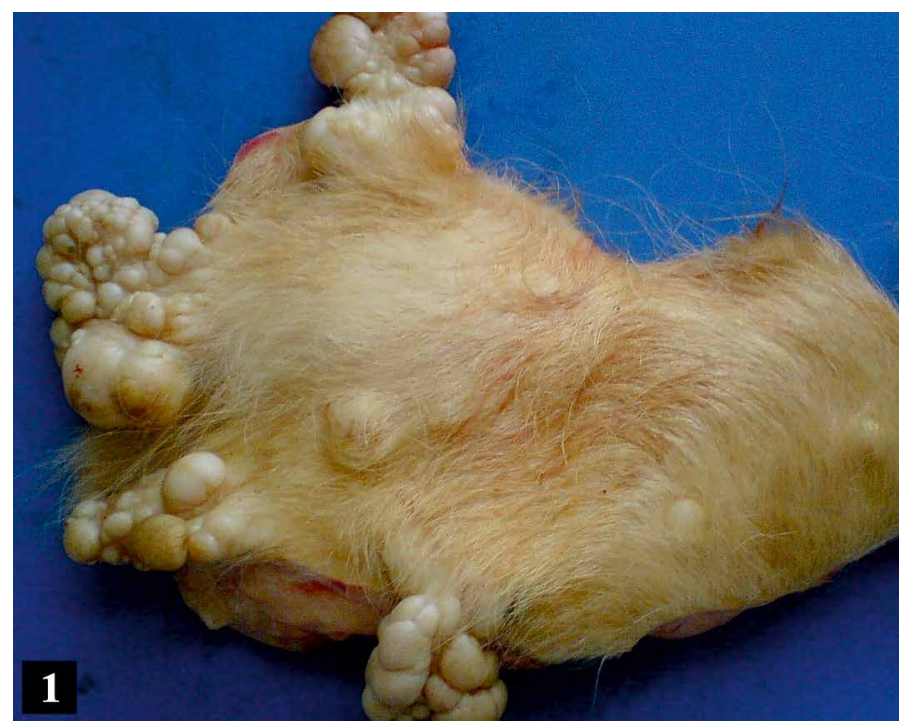

Fig. 1. Macroscopical cauliflower like appearence of papillomatosis from abdominal skin, 3 years old Holstein cow 


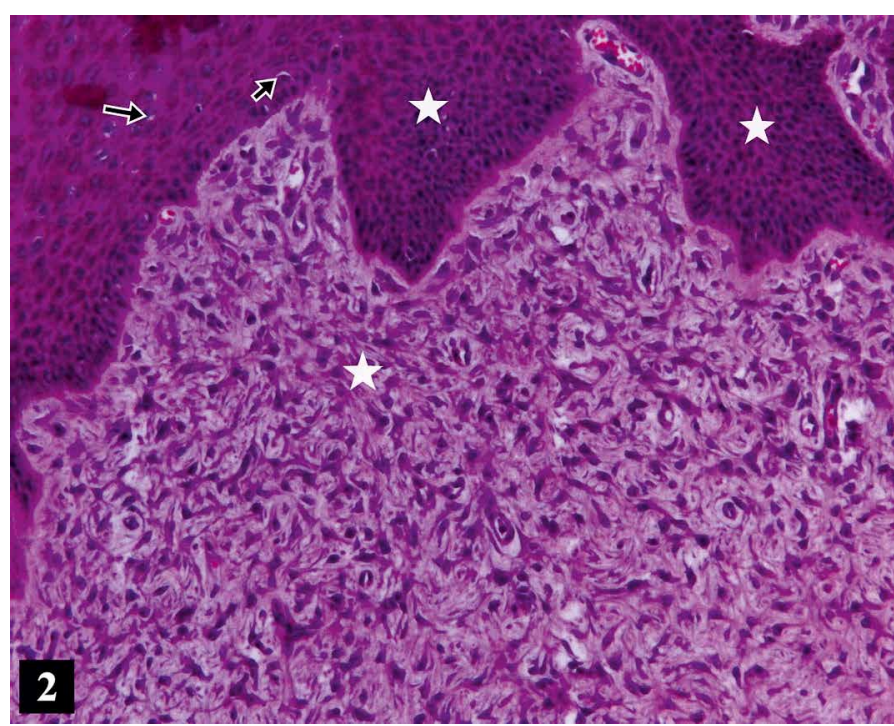

Fig. 2. The fibropapilloma (asteriks) and vacuolar degeneration in stratum spinosum cells (arrows), $\times 100$ objective, $H \& E$

moderate $(5 / 18)$, and strong (1/18) degenerative changes were observed in the remaining cases. The four regressive cases featured fibrocyte and fibroblast proliferation, collagen deposition, and a very thin layer of epidermal cells. Proliferation was more evident in fibropapilloma cases than in papilloma cases. These cases exhibited mild (4/4), moderate (2/4), or strong $(4 / 4)$ increases in the presence of connective tissue components. Mallory's trichrome staining, which was used to confirm proliferation, revealed the presence of neutrophils, lymphocytes, macrophages, and plasma cells (Fig. 2 and 3). These infiltrating leukocytes were present at different proportions and densities among the cases. In all 22 cases, the inflammatory reactions were classified as mild $(6 / 22)$, moderate $(7 / 22)$, or strong $(5 / 22)$. The Feulgen reaction revealed possible viral components in the basal cells of papilloma cases and fibrocytes or fibroblasts of fibropapilloma cases.

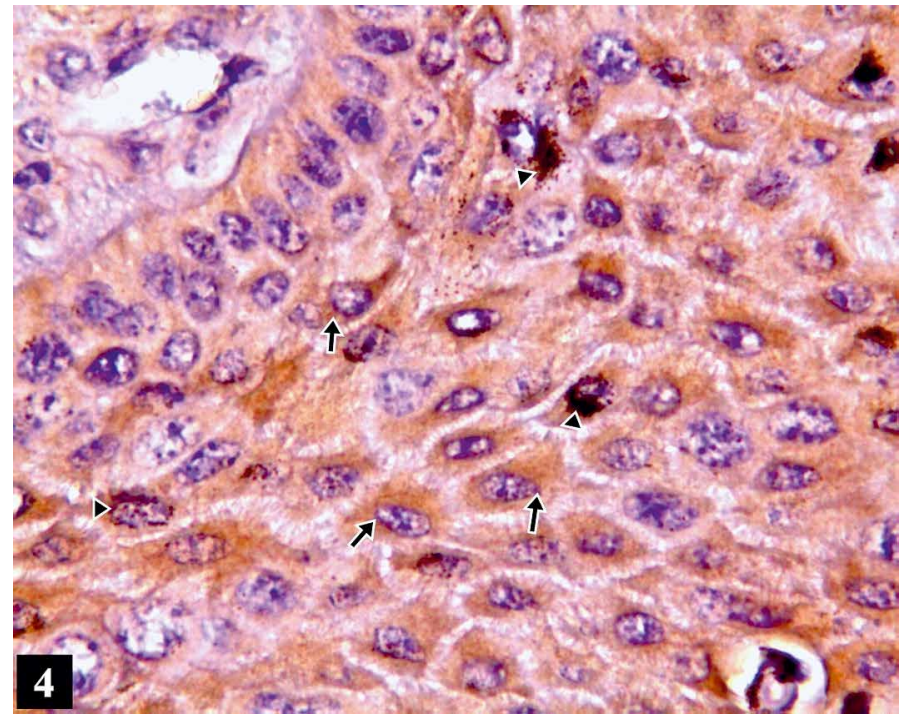

Fig. 4. Cytoplasmic (arrows) and perinuclear BPV-1 positivities (arrow heads) in stratum spinosum cells, $\times 400$ objective, ABC-P

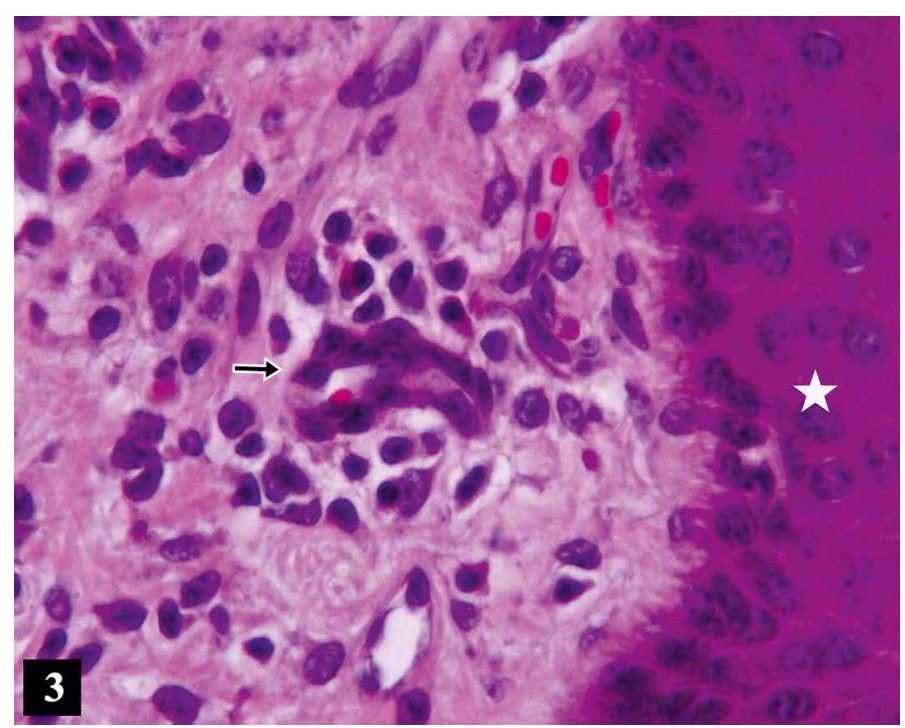

Fig. 3. Mononuclear cell infiltration comprising lymphocyte, plasma cell and macrophage in dermis (arrow) and acantosis (asteriks), $\times 400$ objective, $\mathrm{H \& E}$

Immunohistochemical findings. All samples exhibited a homogenous or granular dark brown reaction with BPV-1 staining. The stratum basale cells exhibited mild (4/22), moderate $(12 / 22)$, or strong reactions $(6 / 22)$, as did the stratum granulare cells [mild (6/22), moderate (6/22), and strong reactions (2/22)]. Except for two negative cases, the stratum spinosum cells had reactions from downside: mild (6/22), moderate (10/22), and strong (4/22); upside: mild (7/22), moderate $(9 / 22)$, and strong reactions $(4 / 22)$. The stratum lucidum reactions were mild $(4 / 22)$, moderate $(4 / 22)$, or strong (1/22), with 13 negative reactions. The stratum corneum reactions were mild $(2 / 22)$ or moderate $(5 / 22)$. Fibrocytes and fibroblasts had mild $(5 / 22)$ and moderate $(5 / 22)$ or strong $(4 / 22)$ reactions. Collagen bundles, where present, were mildly $(3 / 22)$ or moderately (7/22) positive (Fig. 4).

TUNEL apoptosis staining yielded the following findings. The stratum basale were mildly $(5 / 22)$, moderately $(12 / 22)$, or strongly positive $(2 / 22)$. The lower layers of the stratum spinosum were mildly $(6 / 22)$, moderately $(8 / 22)$, or strongly positive $(6 / 22)$. The upper layers were mildly (4/22), moderately (10/22), or strongly $(5 / 22)$ positive. The stratum granulare reactions were mild $(6 / 22)$, moderate $(9 / 22)$, or strong $(3 / 22)$. The stratum lucidum reactions were mild (6/22), moderate $(7 / 22)$, or strong $(2 / 22)$; similarly, reactions in the stratum corneum were mild $(7 / 22)$, moderate $(5 / 22)$, or strong $(1 / 22)$. However, the proportions of fibroblasts and fibrocytes that reacted mildly, moderately, or strongly $(3 / 22,4 / 22$, and $11 / 22$, respectively) differed from the proportions of collagen bundles with similar reactions $(5 / 22,6 / 22$, and $4 / 22$, respectively) (Fig. 5),

CISH findings. E2 gene - The stratum basale reacted mildly $(6 / 22)$, moderately $(9 / 22)$, or strongly $(5 / 22)$. Regarding the stratum spinosum, the lower layers reacted mildly and moderately $(7 / 22)$ or strongly 


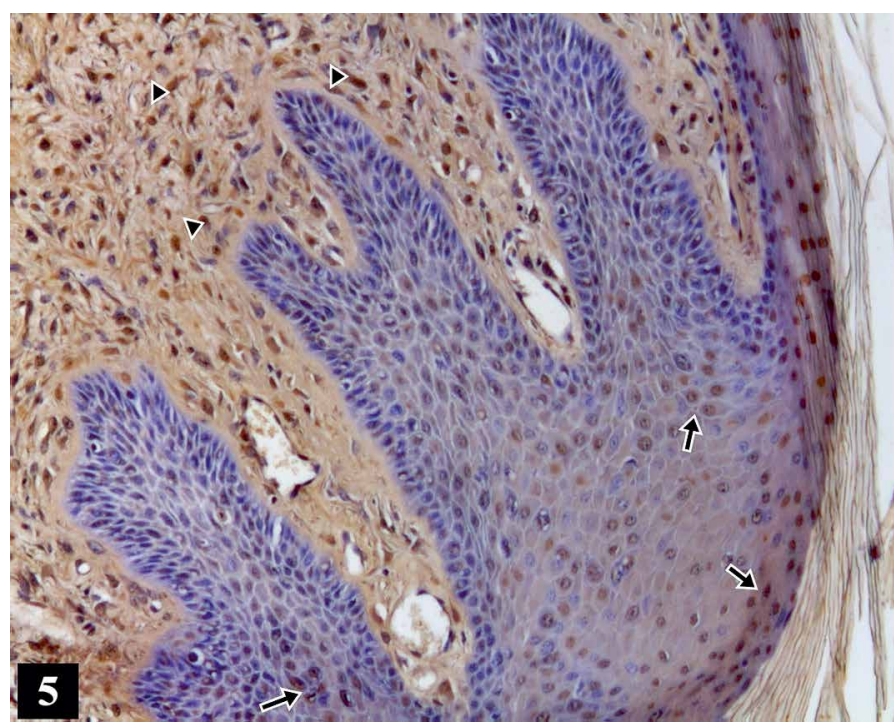

Fig. 5. Apoptosis in stratum spinosum and upper layer cells (arrows) neoplastic fibrocytes and fibroblasts in dermis (arrow heads) in a case of progressive fibropapilloma, $\times 100$ objective, TUNEL

$(3 / 22)$; similarly, the upper layers were mildly and moderately $(6 / 22)$ or strongly $(3 / 22)$ positive. The stratum granulare were mildly $(5 / 22)$, moderately $(6 / 22)$, or strongly $(2 / 22)$ positive. Similar to the previous layer, the stratum lucidum reacted mildly and moderately $(5 / 22)$ or strongly $(2 / 22)$. The stratum corneum was mildly (6/22), moderately, or strongly (3/22) positive (Fig. 6). Fibroblasts and fibrocytes were mildly or moderately $(7 / 22)$ positive. All collagen bundles were negative in all cases.

E5 gene - The stratum basale reacted mildly (7/22), moderately $(7 / 22)$, or strongly $(8 / 22)$. Reactions observed in the stratum spinosum down layers were mild $(8 / 22)$, moderate $(7 / 22)$, and strong $(6 / 22)$, but these numbers differed slightly in the upper layers (mild or moderate, $7 / 22$; strong, 5/22). The stratum granulare reacted mildly, moderately $(6 / 22)$, or strongly (4/22). The stratum lucidum reactions were mild,

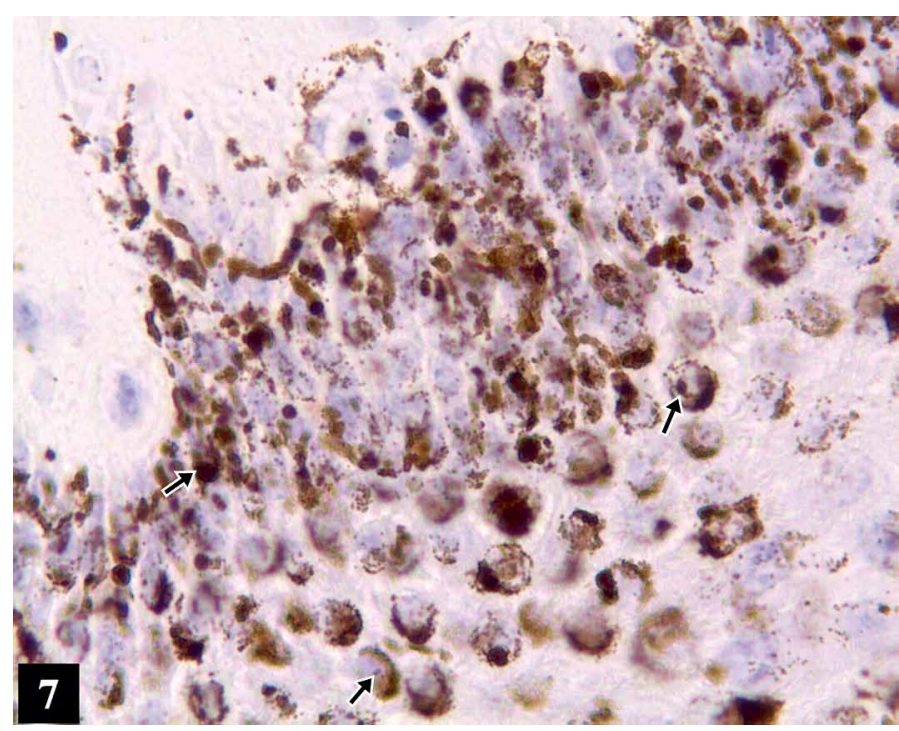

Fig. 7. E5 positivities as nuclear (arrows) in all layer cells of a case of progressive fibropapilloma, $\times \mathbf{4 0 0}$ objective, CISH

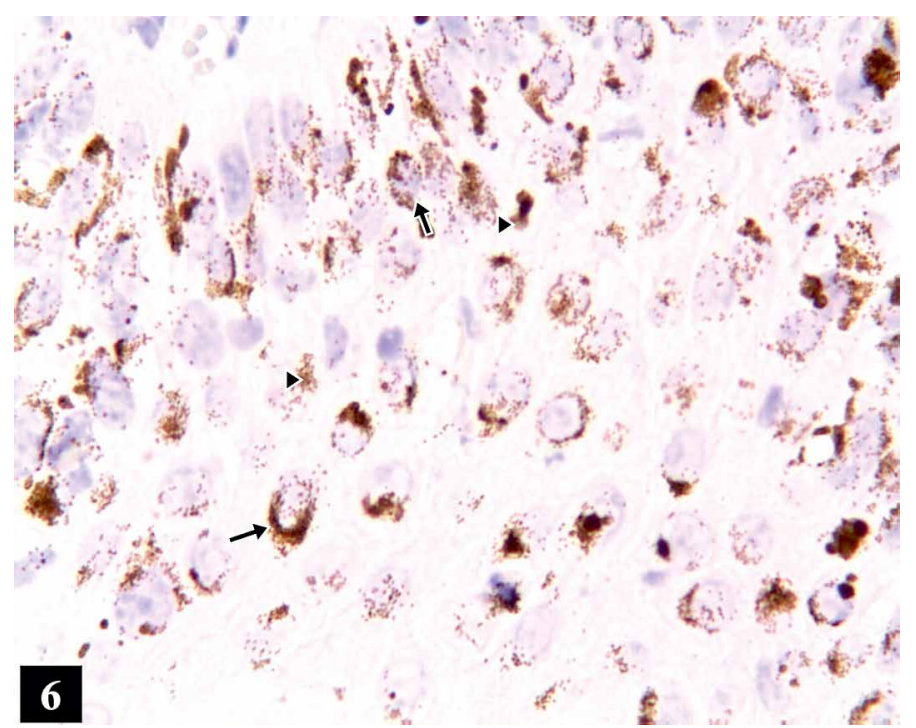

Fig. 6. E2 positivities as perinuclear (arrows) and cytoplasm (arrow heads) in a case of progressive fibropapilloma, $\times 400$ objective, CISH

strong $(4 / 22)$, or moderate $(6 / 22)$. The stratum corneum reactions were also mild, strong $(3 / 22)$, or moderate $(7 / 22)$ (Fig. 7). However, the fibroblasts and fibrocytes were only mild $(9 / 22)$ or moderate $(3 / 22)$. All collagen bundles were negative in all cases.

E6 gene - The stratum basale reacted mildly (7/22), moderately $(4 / 22)$, or strongly $(10 / 22)$. For the stratum spinosum, the lower layers were mild, strong (6/22), or moderate $(7 / 22)$, whereas the upper layers were mild $(6 / 22)$, moderate, or strong (4/22). Similar to the previous layer, the stratum granulare exhibited mildly (4/22), moderately $(5 / 22)$, and strongly $(5 / 22)$ positive reactions. For the stratum lucidum, the reactions were more distributed as mild $(7 / 22)$, moderate $(4 / 22)$, or strongly $(6 / 22)$. The stratum corneum reactions were mild $(5 / 22)$, moderate $(3 / 22)$, or strong $(10 / 22)$. However, the fibroblast and fibrocytes reacted mildly or strongly (3/22) (Fig. 8). Notably, some col-

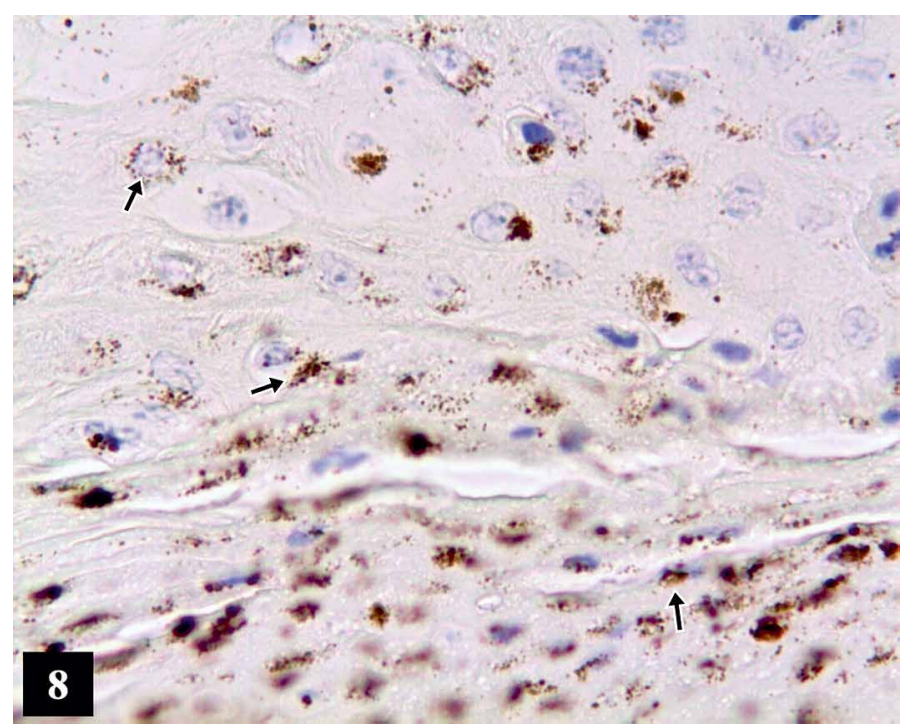

Fig. 8. E6 positivities as nuclear and cytoplasmic in all layer cells a case of regressive fibropapilloma, $\times \mathbf{4 0 0}$ objective, $\mathrm{CISH}$ 
lagen bundles exhibited mild or moderate reactivity $(2 / 22)$.

All immunohistochemical (immunoperoxidase and TUNEL staining) and CISH results corresponding to progressive and regressive changes are presented in Tab. 3-4 and Fig. 10-11.

RT-PCR. The genes were positive at 170 base pairs (bp) (E2), $75 \mathrm{bp}$ (E5), and $119 \mathrm{bp}$ (E6), respectively (Fig. 9).

BPV-1 is currently the best-known etiologic agent of cutaneous papilloma and fibropapilloma in cattle $(5,14)$. In papillomavirus-induced benign neoplastic changes, the virus is localized in cells of the stratum basale and stratum spinosum. During infection, viral early genes (E2, E5, and E6) are expressed from the early region of the viral genome (18). However, viralhost cell chromatin interactions are possibly initiated by modifications in histone conformation that lead to viral DNA integration in the host genome and/or chromosomal rearrangements during proliferation and encoding. These events are considered cellular alterations that collectively may cause oncogenic transformation (3). In this context, E2 genes, which encode viral DNA replication proteins, are expressed initially upon viral entry into the host cell (20). The E5 gene also encodes a viral replication protein that

Tab. 3. The evaluation of immunohistochemical and chromogenic in situ hybridization findings in progressive and regressive changes in epidermis and dermis on the basis of positive cases

\begin{tabular}{|l|c|r|r|r|r|r|r|r|l|l|l|l|l|l|l|l|l|}
\hline Progressive & SB & SS & SG & SL & SC & Fb & F & C & Regressive & SB & SS & SG & SL & SC & Fb & F & C \\
\hline BPV-1 & 18 & 16 & 11 & 8 & 5 & 11 & 11 & 9 & BPV-1 & 4 & 4 & 3 & 2 & 2 & 2 & 2 & 1 \\
Apoptosis & 16 & 16 & 14 & 12 & 9 & 14 & 14 & - & Apoptosis & 3 & 4 & 4 & 3 & 3 & 3 & 3 & - \\
E2 & 16 & 14 & 9 & 8 & 8 & 6 & 6 & 0 & E2 & 4 & 4 & 4 & 4 & 4 & 1 & 1 & 0 \\
E5 & 18 & 17 & 14 & 11 & 10 & 10 & 8 & 0 & E5 & 4 & 4 & 3 & 3 & 3 & 2 & 1 & 0 \\
E6 & 17 & 15 & 10 & 13 & 14 & 5 & 5 & 2 & E6 & 4 & 4 & 4 & 4 & 4 & 2 & 2 & 1 \\
\hline
\end{tabular}

Explanations: SB - stratum basale; SS - stratum spinosum; SG - stratum granulare; SL - stratum lucidum; $\mathrm{SC}$ - stratum corneum; $\mathrm{Fb}$ - fibroblast; $\mathrm{F}$ - fibrocyte; $\mathrm{C}$ - collagen

Tab. 4. Percentage (\%) of all reactions belonging to immunohistochemistry and chromogenic in situ hybridization (CISH)

\begin{tabular}{|c|c|c|c|c|c|c|c|}
\hline $\begin{array}{l}\text { Degree of } \\
\text { Positivities }\end{array}$ & Str. basale & Str. spinosum & Str. granulosum & Str. lucidum & Str. corneum & $\begin{array}{l}\text { Fibrocyte- } \\
\text { Fibroblast }\end{array}$ & Collagen Bundle \\
\hline \multicolumn{8}{|l|}{ Negative } \\
\hline BPV-1 & 0 & 9.10 & 36.36 & 59.10 & 68.18 & 36.36 & 54.54 \\
\hline E2 gene & 9.10 & 22.73 & 40.90 & 45.45 & 45.45 & 36.36 & 100 \\
\hline E5 gene & 0 & 4.56 & 27.27 & 36.36 & 40.90 & 45.45 & 100 \\
\hline E6 gene & 4.54 & 13.64 & 36.36 & 4.54 & 18.18 & 72.72 & 81.82 \\
\hline TUNEL react. & 13.63 & 9.09 & 18.18 & 31.82 & 40.91 & 18.18 & 31.82 \\
\hline \multicolumn{8}{|l|}{ Mild } \\
\hline BPV-1 & 18.18 & 31.81 & 27.27 & 18.18 & 9.10 & 22.72 & 13.64 \\
\hline E2 gene & 27.27 & 31.18 & 22.72 & 22.73 & 27.27 & 31.82 & 0 \\
\hline E5 gene & 31.82 & 36.36 & 27.27 & 18.19 & 13.64 & 40.90 & 0 \\
\hline E6 gene & 31.81 & 27.27 & 18.18 & 31.18 & 22.73 & 13.64 & 9.09 \\
\hline TUNEL react. & 22.73 & 22.73 & 27.27 & 27.27 & 31.82 & 13.64 & 22.73 \\
\hline \multicolumn{8}{|l|}{ Moderate } \\
\hline BPV-1 & 27.27 & 45.45 & 27.27 & 18.18 & 22.72 & 22.72 & 31.82 \\
\hline E2 gene & 40.90 & 31.18 & 27.27 & 22.73 & 13.64 & 31.82 & 0 \\
\hline E5 gene & 31.82 & 31.81 & 27.27 & 18.19 & 31.32 & 13.65 & 0 \\
\hline E6 gene & 18.18 & 27.27 & 22.72 & 18.18 & 13.64 & 0 & 9.09 \\
\hline TUNEL react. & 54.54 & 40.91 & 40.91 & 31.82 & 22.73 & 18.18 & 27.27 \\
\hline \multicolumn{8}{|l|}{ Strong } \\
\hline BPV-1 & 54.54 & 13.64 & 9.10 & 4.54 & 0 & 18.20 & 0 \\
\hline E2 gene & 22.72 & 13.64 & 9.10 & 9.09 & 13.64 & 0 & 0 \\
\hline E5 gene & 36.36 & 27.27 & 18.20 & 27.27 & 13.64 & 0 & 0 \\
\hline E6 gene & 45.45 & 31.82 & 22.72 & 27.27 & 45.45 & 13.64 & 0 \\
\hline TUNEL react. & 9.09 & 27.27 & 13.64 & 9.09 & 4.54 & 50.00 & 18.18 \\
\hline
\end{tabular}



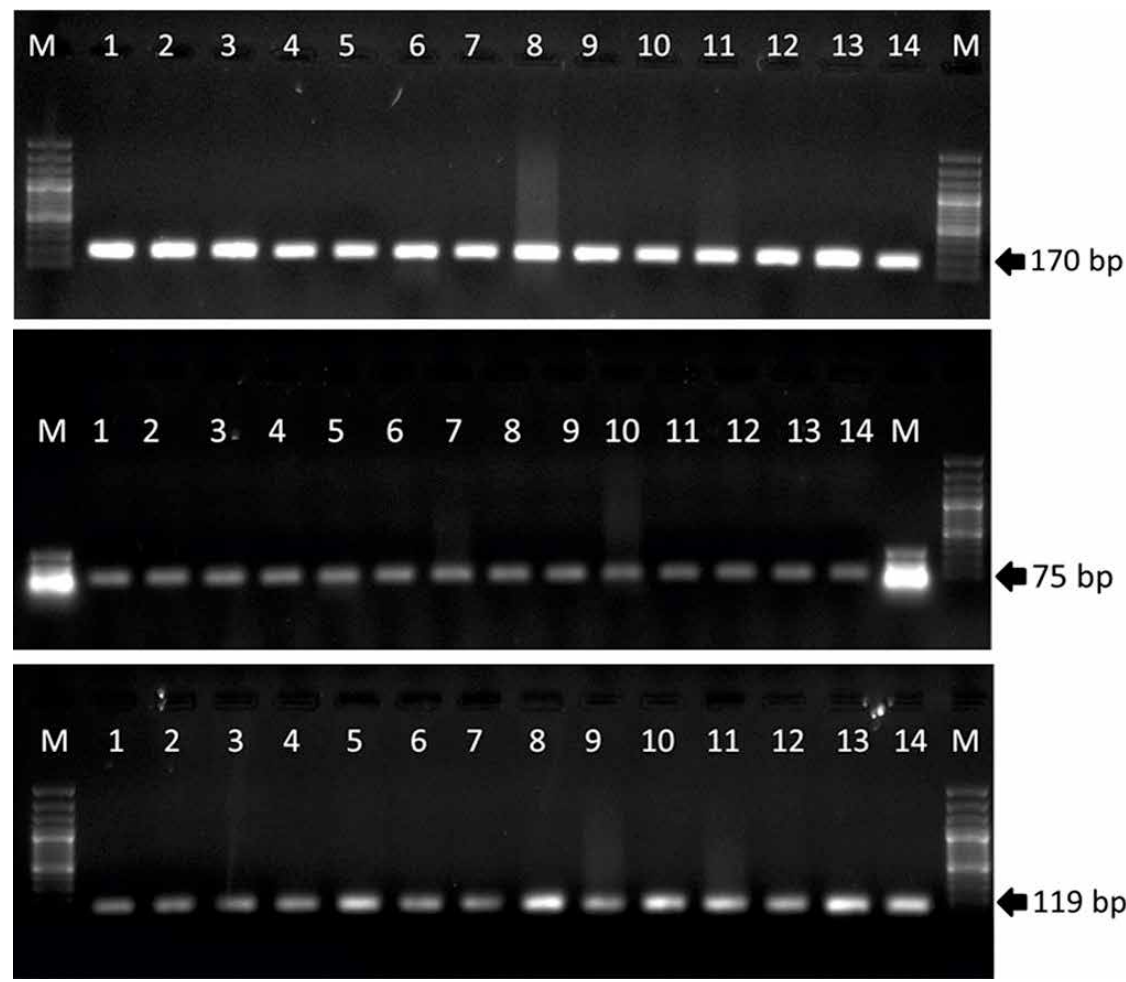

Fig. 9. RT-PCR for BPV-1, E2, E5 and E6 gene results

Explanation: M - Marker (100 bp DNA ladder, Thermo Scientific, Germany) growth during the progressive phase. In our study, we observed that during the progressive phase, BPV-1 distribution and E2 and E5 gene expression decreased by approximately one-third from the stratum basale toward the stratum corneum. However, none of the genes were effectively expressed in connective tissue compartments.

A previous report on E5 localization described similar findings regarding transformed epithelial cells from buffalo fibropapillomas during the early stages of viral infection; namely, E5 was expressed strongly in the basal and granular layers and sporadically in the spinous cell layer $(1,13)$. In addition, strong E5 expression was observed in keratinocytes $(2,4)$. In contrast to these earlier findings, the basal-granular and spinosum cell layers from both progressive and regressive lesions had generally moderate or strong positive E5 positivity, despite the more moderate reactions in the upper layer cells and keratinocytes. On the other assists with viral DNA replication and is abundantly expressed in some differentiated tumor epithelial cells that contain and can shed virions (23). In other words, both genes and oncoproteins play pivotal roles in tumor hand, previous studies of fibropapilloma have reported E5 expression in the nuclear and perinuclear regions (particularly the Golgi apparatus) in comparisons of basal and superficial epithelial cells and fibroblasts. Our study identified similar patterns of E5 positivity. Accordingly, E5 expression is believed to play a role in promoting the growth of all tumor components from deep to surface layers.

BPV-1 encodes E6 oncoproteins excepting E2 and E5 are the major transforming protein interacting with each other $(16,21)$. E6 is another crucial factor in this transformation. Similar to the other genes, E6 is expressed mainly in virus-infected basal and suprabasal cells and rarely in spinosum cells (19). E6 might promote transformation through

Fig. 10. Distribution of the changes encountered in papilloma and fibropapilloma

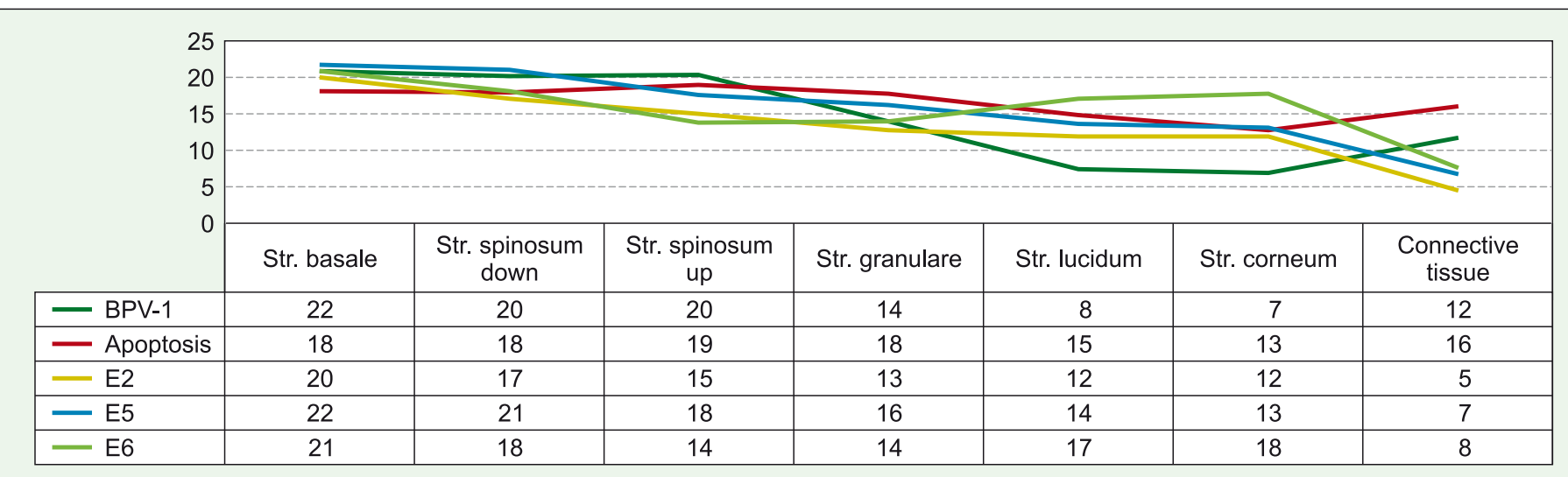

Fig. 11. Comparing of all immunoperoxidase, TUNEL and CISH positivities 
its interaction with the focal adhesion protein paxillin (28). The combined effect of the E5 and E6 genes has been described as crucial for the growth and invasion of sarcoid fibroblasts, another type of BPV-1-indcued tumor. Thus, the inhibition of these viral oncoproteins would be expected to impair cell growth and invasion (29). In our study, the E5 gene had an effect on both connective tissue components and epithelial cells. In contrast to our expectations, however, the E6 gene was less effective in the regressive phase. This finding suggests that the E6 gene only participates in oncogenic transformation during the progressive phase.

The E5 and E6 oncoproteins have also been reported to have an interactive effect on cells from HPV-related cancers (19). Thus, it is possible that the combined role of E5 and E6 changes in the context of tumor regression. Regarding the relationship between apoptosis and the E6 oncoprotein, in HPV16, the E6 oncoprotein acts a transcription factor to trigger cell cycle arrest or apoptosis in response to a large variety of cellular stresses, such as hypoxia or DNA damage $(13,22)$.

In some situations, the E2 oncoprotein might also be required for the regression of endogenous HPV genes (7). Accordingly, E2 proteins can elicit HPV-independent effects such as apoptosis. Furthermore, previous studies found that transfection of the BPV and HPV18 E2 genes could induce apoptosis by arresting p53-dependent growth $(9,10,27)$. According to these findings, E2 is considered to play a bilateral role in regression-phase situations. However, it has been mentioned that the fate of progression or regression in a papilloma or fibropapilloma rests on the presence and roles of various molecules. In our study, E2 gene expression and apoptosis were reduced in only a quarter of samples in the progressive phase. In the regressive phase, the decreases in E2 gene and apoptosis were even less apparent.

The pathomorphological, immunohistochemical, and molecular findings of the present study indicated that 18 samples were progressive (eight in the growth phase, 10 in the development phase), whereas four were regressive. Of the progressive cases, 14 were fibropapilloma and three were papilloma. Of the regressive cases, three were fibropapilloma and one was papilloma. In the progressive phase cases, the distribution of BPV-1 and expression of E2 and E5 genes decreased by one-third from the stratum basale toward the corneum; this decrease was two-thirds for E6 gene expression and apoptosis. BPV-1 distribution was reduced in fibroblasts, fibrocytes, and collagenous deposits from half of the samples: in these components, E gene expression was reduced in a third of samples, and apoptosis marker expression was reduced in two thirds; however, in epithelial layers from regressivephase lesions, BPV-1 distribution was reduced in approximately half of the samples, whereas E gene expression and apoptosis were reduced in a quarter of samples. Similarly, BPV-1, E5, and E6 gene expression were decreased in half of the evaluated fibroblasts, fibrocytes, and collagen deposits, whereas E2 gene expression and apoptosis were decreased only in a quarter of samples. Although E5 and E6 might have an overall regressive effect on neoplastic cells, this should be evaluated in the context of other p53 pathway and apoptotic cascade signals.

Morphological changes in skin and anti-viral host reactions have been reported to regress following the progressive phase (15). However, no meaningful reports have investigated the role of the most critical E genes and their expression levels in papilloma and fibropapilloma. This study is therefore expected to clarify the issues surrounding this subject.

In conclusion, most reports of BPV-1 cases describe the virological aspects, despite the presence of pathomorphological and immunohistochemical studies $(2,4$, $6,8,11,23)$. However, some studies have demonstrated relationships between other E genes and apoptosis (16). To the best of our knowledge, previous reports have not mentioned bilateral trends in E gene expression with respect to progression and regression. Furthermore, earlier reports have not documented the distribution of apoptosis in components of the dermis and epidermis during the regression and progression phases of natural papillomas and fibropapillomas. Natural BPV-1 infection is expected to shed light and provide details on the basis of the progressive/regressive classification. Accordingly, the authors believe that the current study provides a significant contribution to research involving the molecular and immunopathological features of BPV-1 infection.

\section{References}

1. Anderson R. A., Scobie L., O'Neil B. W., Grindlay G. J., Campo M. S.: Viral proteins of bovine papillomavirus type 4 during the development of alimentary canal tumors. Vet. J. 1997, 154, 69-78.

2. Bohl J., Hull B., Vande Pol S. B.: Cooperative Transformation and Coexpression of Bovine Papillomavirus Type 1 E5 and E7 Proteins. J. Virol. 2001, 75, 513 -521 .

3. Borzacchiello G., Roperto F.: Bovine papillomaviruses, papillomas and cancer in cattle. Vet. Res. 2008, 39, 45.

4. Burnett S., Jareborg N., DiMaio D.: Localization of bovine papillomavirus type 1 E5 protein to transformed basal keratinocytes and permissive differentiated cells in fibropapilloma tissue. Proc. Natl. Acad. Sci. USA 1992, 89, 5665-5669.

5. Campo M. S.: Bovine papillomavirus and cancer. Vet. J. 1997, 154, 175-188. 6. Claus M. P., Lunardi M., Alfieri A. A., Otonel R. A. A., Sartori D., Helena M., Fungaro P., Alfieri A. F.: Multiple bovine papillomavirus infections associated with cutaneous papillomatosis in brazilian cattle herds. Braz. Arch. Biol. Technol. 2009, 52, 93-98.

7. DeFilippis R. A., Goodwin E. C., Wu L., DiMaio D.: Endogenous human papillomavirus E6 and E7 proteins differentially regulate proliferation, senescence, and apoptosis in HeLa cervical carcinoma cells. J. Virol. 2003, 77, 1551-1563.

8. DeMasi J., Huh K. W., Nakatani Y., Münger K., Howley P. M.: Bovine papillomavirus E7 transformation function correlates with cellular p600 protein binding. Proc. Natl. Acad. Sci. USA 2005, 102, 11486-11491.

9. Desaintes C., Demeret C., Goyat S., Yaniv M., Thierry F.: Expression of the papillomavirus E2 protein in HeLa cells leads to apoptosis. EMBO J. 1997, $16,504-514$.

10. Desaintes C., Goyat S., Garbay S., Yaniv M., Thierry F.: Papillomavirus E2 induces p53-independent apoptosis in HeLa cells. Oncogene 1999, 18, 4538-4545 . 
11. Gillette T. G., Borowiec J. A.: Distinct roles of two binding sites for the bovine papillomavirus (BPV) E2 transactivator on BPV DNA replication. J. Virol. 1998, 72, 5735-5744.

12. Goldschmidt M. H., Hendrick M. J.: Tumors of the skin and soft tissues, [in:] Meuten D. J., (ed.), Tumors in Domestic Animals., Iowa State Press, Ames, USA 2002, p. 47-117.

13. Huibregtse J. M., Scheffner M., Howley P. M.: A cellular protein mediates association of $\mathrm{p} 53$ with the E6 oncoprotein of human papillomavirus types 16 or 18 . EMBO J. 1991, 10, 4129.

14. Jelinek F., Tachezy R.: Cutaneous papillomatosis in cattle. J. Comp. Pathol. 2005, 132, 70-81.

15. Kolodzieyskj L., Revajova V.: Distribution of CD3 lymphocytes in the lymph nodes of cows infected by BL V. Folia Veterinaria 1996, 40, 61-64.

16. Liu Y., Liu Z., Gao H., Zhou Y., Androphy E. J., Chen J. J.: Opposing effects of bovine papillomavirus type 1 E6 and E7 genes on Fas-mediated apoptosis. Oncogene 2005, 24, 3942-3953.

17. Liu Z., Liu Y., Hong Y., Rapp L., Androphy E. J., Chen J. J.: Bovine papillomavirus type 1 E6-induced sensitization to apoptosis is distinct from its transforming activity. Virology 2002, 295, 230-237.

18. Middleton K., Peh W., Southern S. A., Griffin H. M., Sotlar K., Nakahara T., Sherif A. E., Morris L., Seth R., Hibma M., Jenkins D., Lambert P., Coleman N., Doorbar J.: Organisation of the human papillomavirus productive cycle during neoplastic progression provides a basis for the selection of diagnostic markers. J. Virol. 2003, 77, 10186-10201.

19. Moody C. A., Laimins L. A.: Human Papillomavirus Oncoprotein: Pathways to Transformation. Nature Reviews. Cancer 2010, 10, 550-560.

20. Motoyama S., Ladines-Llave C. A., Luis Villanueva S., Maruo T.: The role of human papilloma virus in the molecular biology of cervical carcinogenesis. Kobe. J. Med. Sci. 2004, 50, 9-19.

21. Petti L., DiMaio D.: Specific interaction between the bovine papillomavirus E5 transforming protein and the beta receptor for platelet-derived growth factor in stably transformed and acutely transfected cells. J. Virol. 1994, 68, 3582-3592.

22. Scheffner M., Werness B. A., Huibregtse J. M., Levine A. J., Howley P. M.: The E6 oncoprotein encoded by human papillomavirus types 16 and 18 promotes the degradation of p53. Cell 1990, 63, 1129.

23. Silvestre O., Borzacchiello G., Nava D., Iovane G., Russo V., Vecchio D. D'Ausilio F., Gault E. A., Campo M. S., Paciello O.: Bovine papillomavirus type 1 DNA and E5 oncoprotein expression in water buffalo fibropapillomas. Vet. Pathol. 2009, 46, 636-641.

24. Sundberg J. P.: Papillomavirus infections in animals, [in:] Syrjänen K., Gissmann L., Koss L. (eds.): Papillomaviruses and human disease. SpringerVerlag, Heidelberg, Germany 1987, p. 40-103.

25. Sundberg J. P., Phillips G., O’Brien S. J., Jenson A. B., Burk R. D., Ranst M. $V$ : Papillomavirus- associated focal oral hyperplasia in wild and captive asian lions (panthera leo persica). J. Zoo Wild Med. 1996, 27, 61-70.

26. Suprynowicz F. A., Baege A., Sunitha I., Schlegel R.: c-Src activation by the E5 oncoprotein enables transformation independently of PDGF receptor activation Oncogene 2002, 21, 1695-1706.

27. Thierry F., Yaniv M.: The BPV1-E2 trans-acting protein can be either an activator or a repressor of the HPV18 regulatory region. EMBO J. 1987, 6, 3391-3397.

28. Tong X., Howley P. M.: The bovine papillomavirus E6 oncoprotein interacts with paxillin and disrupts the actin cytoskeleton. Proc. Natl. Acad. Sci. USA 1997, 94, 4412-4417.

29. Yuan Z., Gault E. A., Campo M. S., Nasir L.: Different contribution of bovine papillomavirus type 1 oncoproteins to the transformation of equine fibroblasts. J. Gen. Virol. 2011, 92, 773-783.

Corresponding author: Dr. Mehmet Eray ALCIGIR, phD, Ankara University, Faculty of Veterinary Medicine, Department of Pathology, 06110, Diskapi-Ankara/Turkey; e-mail: erayalcigir@gmail.com, ealcigir@ankara.edu.tr 